\title{
Different Optical Methods for Clinical Monitoring of the Microcirculation
}

\author{
Frank Christ Andreas Bauer Dirk Brügger \\ Department of Anesthesiology, Ludwig Maximilians University Munich, Germany
}

\section{Key Words}

Monitoring · Intravital microscopy · Laser Doppler fluxmetry · Near-infrared spectroscopy · Orthogonal polarisation spectral imaging

\section{Introduction}

In the past perioperative as well as critically ill patient care monitoring has very much focused on normalizing macrocirculatory indices of patient well being. Recent research, however, suggests that in particular changes in the microcirculation are responsible for the deterioration of cell and organ function. Pathological events at the microcirculatory interface are responsible for severe morbidity as well as mortality from some of the most common causes of perioperative death, such as sepsis, acute respiratory distress syndrome, and multi-organ failure syndrome [1-3]. To improve the somewhat stagnant figures of patient outcome after most of these diseases it seems important to readdress what we should monitor in patients. Focusing on the microcirculation seems a very desirable approach leaving us, however, with the question what should and what can we currently monitor. The following short review focusses on some of the available optical techniques for microcirculatory patient monitor- ing, since they are noninvasive and usually easy to apply. We will try to give an account of the clinical value of the techniques and the future potentials.

\section{Laser Doppler Fluxmetry}

In laser Doppler fluxmetry (LDF) monochromatic laser light is emitted into the tissue. The frequency broadening of the backscattered light as a result of the interaction of laser light with moving red blood cells was first described by Stern in 1975 [4]. Processing of this signal to a linear output reflecting changes in microvascular blood flow was subsequently used to develop the laser Doppler fluxmetry [5]. LDF utilises the Doppler shift to give a value of the mean erythrocyte flux found in the investigated tissue.

After flow occlusion in vivo, an LDF flow greater than 0 is usually observed. Tenland et al. [6] suggested that this signal should be called 'biological zero' and subtracted from the total output signal. The origin of this background noise remains unknown, but studies suggest that it arises from residual vasomotion and Brownian motion of the macromolecules within the interstitium [7]. The biological zero should always be measured and subtracted from the flow signal given in experimental and clinical conditions.

\begin{tabular}{ll}
\hline KARGER & ( ) 2002 S. Karger AG, Basel \\
Fax +41613061234 & $0014-312 X / 02 / 0342-0145 \$ 18.50 / 0$ \\
$\begin{array}{l}\text { E-Mail karger@karger.ch } \\
\text { www.karger.com }\end{array}$ & $\begin{array}{l}\text { Accessible online at: } \\
\text { www.karger.com/journals/esr }\end{array}$
\end{tabular}

Dr. Frank Christ, MD
Department of Anesthesiology, Ludwig Maximilians University Munich
Marchioninistrasse 15, D-81366 Munich (Germany)
Tel. +4989 70953416, Fax +49 8974029548
E-Mail frank.christ@ana.med.uni-muenchen.de 
The LDF technology is very easy to apply and requires only a few minutes to obtain readings. However, the probe has to be attached to the tissue and movement of the fibre optic during measurements can result in artifacts that may make difficult the interpretation of the results thus limiting some applications.

LDF measures the temporal variation of tissue perfusion in a very small area. Large spatial and temporal fluctuation in tissue blood flow occur over small areas of skin and, thus, influence the LDF signal [8-10]. Furthermore, the depth of penetration of the laser light is limited to a few millimeters and since the signal originates mainly from the arterio-venous anastomosis in the skin it does not reflect the nutritious blood flow of the skin.

Though a linear correlation between LDF signal and blood flow could be demonstrated [4, 5], no calibration for absolute values is currently available, which very much restricts the clinical use of LDF.

Nevertheless, LDF has been used to study microcirculatory changes in chronic venous insufficiency (CVI) and chronic venous ulceration. Increased laser Doppler flux reflecting elevated subcutaneous blood flow could be correlated with worsening clinical symptoms of CVI [11]. Furthermore, by LDF perfusion was found to be increased in the skin around a venous leg ulcer [12].

Ubbink et al. [13] tested the usefulness of LDF in assessing the severity of lower limb ischemia and concluded that it could be a suitable addition to standard macrocirculatory techniques. However, they found that predicted values of the hyperemic response of the LDF signal were lower than the findings of the conventional ankle/ brachial index, and LDF revealed no significant differences between the degrees of ischemia. LDF has been suggested to be a useful microvascular monitor during surgery. Belboul and al-Khaja [14] evaluated the effect of coronary revascularisation on the epicardial microcirculation by LDF and found a positive correlation between the flux signal and blood flow after coronary artery bypass grafting. Dodson et al. [15] assessed gingival blood flow during Le Fort I osteotomy and demonstrated a reduced LDF signal during the course of operation. Furthermore, a longitudinal LDF study suggests that maxillary perfusion can be assessed after this type of operation [16].

Nonlinear, time-variant and higher-order spectral analysis of LDF may provide additional information which cannot be detected with conventional approaches to data analysis [17-19]. The true clinical value of this approach however still has to be proven.
A recent development of LDF is laser Doppler perfusion imaging (LDI) in which a laser beam scans a certain area of the tissue to form an image of perfusion [9]. Conventional LDF records perfusion continuously at one point only. Investigations of a larger area can integrate different local flow readings over a specified area rather than temporal variation at a single point and assesses the spatial distribution of microvascular perfusion. Since LDI is a scanning technique, the probe does not come in contact with the investigated tissue, enabling new applications of the laser Doppler technique.

Recently published studies showed benefits of LDI in assessing the depth of burns $[20,21]$ and in monitoring the activity of rheumatoid arthritis [24]. LDI gave elevated skin perfusion values which were associated with the arthritis of metacarpo-phalangeal joints in patients with active rheumatoid arthritis [22]. LDI values correlated with pain scores and ultrasonographic findings of synovitis. Both studies reflect the initial experiences with this technique but emphasise the potential of LDI in assessing burns and rheumatoid arthritis.

Some methodological flaws also exist in LDI, e.g. the influence of the scanner-to-tissue distance on the perfusion signal. Whereas Kernick and Shore [23] found increased LDI output with higher scanner-to-tissue distance, Droog et al. [21] reported a decreased signal. This discrepancy may be attributed to the use of different imaging equipment. Moreover, increased avascular tissue, dressing and topical agents over the investigated skin reduce the LDI signal [21,23] and have to be considered. Furthermore, the 'biological zero' contributes a similar proportion to the output signal in LDI and LDF and therefore has to be subtracted [23]. As in conventional LDF, LDI can not be calibrated for absolute values, thus, laser Doppler data must be expressed in arbitrary units.

In conclusion, the large spatial and temporal fluctuation of the laser Doppler signal limits the clinical application of this easy to use noninvasive technique. The newly developed LDI scanning device somewhat overcomes this problem, but the lack of absolute values still remains the main problem of LDF techniques for patient monitoring.

\section{Near-Infrared Spectroscopy (NIRS)}

First described by Jöbsis in 1977 [24], this technique allows to monitor the oxygen saturation of various tissues utilizing laser light with wave lengths ranging from the visible to the nonvisible near-infrared light. NIRS gives changes in hemoglobin, reduced hemoglobin, oxidised

\footnotetext{
$\overline{146} \quad \overline{\text { Eur Surg Res 2002;34:145-151 }}$
} 
cytochrome aa3 and total hemoglobin concentration [25]. Initially it was described for fetal and neonatal research. Later NIRS was also utilized in other clinical areas including adult anesthesia and critical care.

\section{Principle of NIRS}

NIRS appears attractive as a monitor of the microcirculation since it penetrates deep tissue segments. The technique is based on the oxygen-dependent near-infrared light absorption properties of hemoglobin and cytochrome aa3. Measurements can be performed either by the transmission or the reflection (backscattering) mode. Since multiple scattering takes place, the optical path length may be several times longer than the physical separation. Quantitative estimates can only be achieved if the relationship between the physical and the optical path length is known. The mean optical path length can be calculated by the mean time-of-flight of photons propagated from a picosecond TiSa laser [25].

\section{Fetal Monitoring}

NIRS measurements were used for noninvasive intrapartum real-time fetal monitoring [26, 27]. It has been shown that NIRS can identify disturbances to total cerebral hemoglobin concentration and cerebral oxygenation that may occur in association with commonly observed problems of hypoxia, e.g. asphyxia during labor. Changes in the concentration of total hemoglobin are of great importance since they are considered to represent changes in cerebral blood volume. In the future, a reliable, noninvasive monitoring of fetal cerebral oxygenation as an alternative to fetal scalp blood sampling may be helpful to decide whether clinical interventions are necessary.

\section{Neonatal Monitoring}

Hypoxic ischemic injury is the most common cause of long-term neurodevelopmental disability in newborn infants who need intensive care. Therefore, noninvasive techniques to examine cerebral oxygenation and hemodynamics may help to prevent damage to the brains of infants. Wyatt et al. [28] designed an apparatus for nearinfrared spectrophotometry and quantified the changes in cerebral oxygenation in sick infants instantaneously at the cot side. Edwards et al. [29] described a cot-side measurement of cerebral blood flow by means of NIRS in ill newborn infants. Despite promising first results this technique is so far not widely used probably due to some methodical flaws (see criticism).

\section{Adult Anesthesia Monitoring}

Reliable early detection of changes in brain oxygenation in patients requiring anesthesia and critical care has been a persistent challenge. Several publications have evaluated the potential of NIRS in adults [30-32]. In patients after head trauma or with increased intracranial pressure the results were rather disappointing, since the correlations established by monitoring of, e.g., increased intracranial pressure, local tissue $\mathrm{PaO}_{2}$ measurements, or jugular vein $\mathrm{HbO}_{2}$ saturation were poor. No clear benefit of NIRS could be shown and a good-quality signal was rather the exception than the rule [33].

Some more recent studies however suggest that NIRS may have a place in intraoperative monitoring when used as an indicator of insufficient oxygen supply during surgery of the carotid artery and during cardiopulmonary bypass [34-37]. As far as monitoring of septic patients is concerned only one study has been published to date, which showed an impaired vascular responsiveness when compared to non-septic critically ill patients [38]. This data is, however, of minor clinical relevance, since it did not offer additional information that could not be obtained with other techniques like plethysmography or LDF.

\section{Criticism}

This technology was initially judged to be very promising due to its noninvasive nature and the potential to give an index of crucially important parameters of patient well being. However, it has not found widespread acceptance since some major setbacks seem to hamper its clinical application. Only relative values can be given, since no calibration mode has been developed to date. As a result no critical level of oxygenation is available which limits the clinical value of NIRS. Measurement of optical path length with time-resolved techniques is not suitable for routine clinical application. Moreover uncertainties exist from which tissue the signal originates [39]. There is clear evidence that near-infrared light with the power used in most commercially available applications of NIRS can penetrate human bone and pick up signals from a depth of several centimeters. Recent results, however, suggest that the main signal originates from rather superficial layers and the aim to quantify changes in oxygen content of brain tissue is not fulfilled. Moreover, changes in interoptode distance during the measurements may occur due to movements or sliding of the probes, changing the optical path length. In addition, the signal-to-noise ratio is poor, and in some studies a useful signal could only be obtained in less than $30 \%$ of the measurements. 


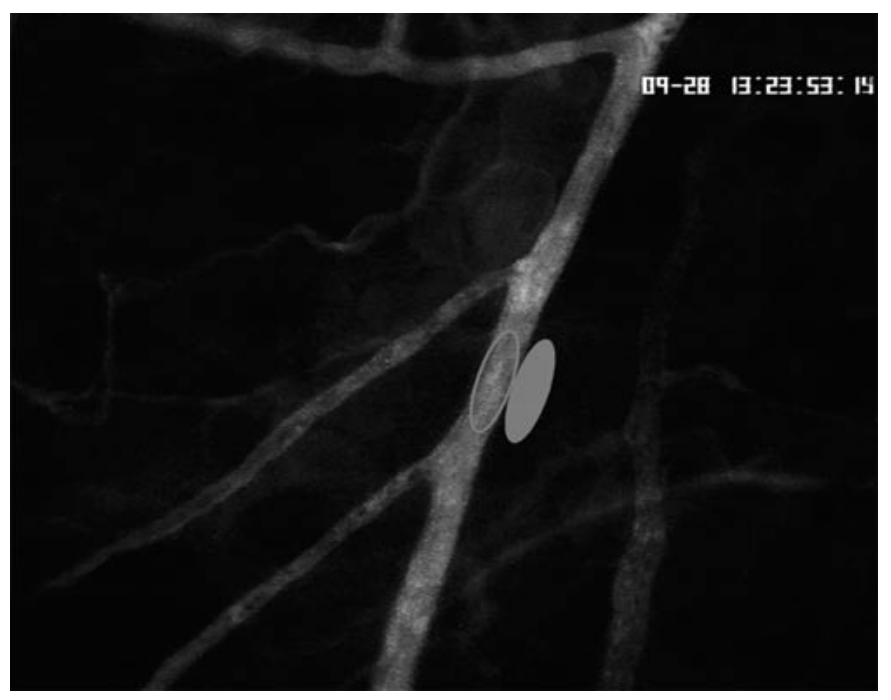

Fig. 1. The intravital microscopy picture was taken from the hamster skin fold chamber model and shows the measurement of macromolecular leakage with FITC labelled dextran around a post capillary venule (with permission from Dr. A. Harris, Institute of Surgical Research, LMU Munich).

\section{Conclusion}

Near-infrared spectroscopy allows noninvasive monitoring of cerebral oxygenation in the fetus, the newborn and the adult. A reliable determination of cytochrome aa3 redox state has not yet been achieved. Unless the signalto-noise ratio is significantly improved and a calibration procedure - and, thus, the possibility to provide a critical value of tissue oxygenation - becomes available, nearinfrared spectroscopy has currently to be judged of minor importance for critically ill patient monitoring. Whether it has a role in perioperative monitoring particularly when cerebral perfusion may be impaired awaits further evaluation.

\section{Intravital Microscopy}

In the research setting intravital microscopy serves as a nearly ideal microcirculatory monitor. In the past 2 decades it has provided us with new information about the microcirculation and has allowed a quantitative assessment of microcirculatory events (thus) improving the pathophysiological understanding of many relevant diseases [40]. Intravital microscopy allows to visualize the interaction of blood components with the endothelium (e.g. leukocytes and thrombocytes) and the leakage of macromolecules into the tissue. As an example figure 1

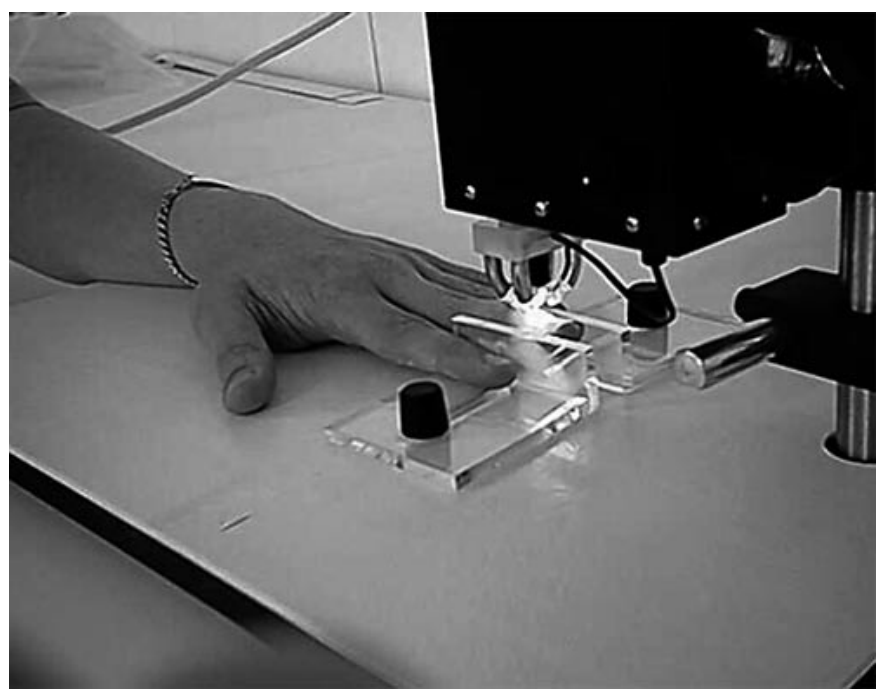

Fig. 2. Example of capillaroscopy applied to the human nailfold (with permission of KK Technologies).

shows the measurement of the leakage of fluoresceinlabeled dextran from a postcapillary venule. Both the leukocyte-endothelium interaction and the increased microcirculatory permeability are important steps in the development of tissue damage and impaired nutritive flow, hence, very worthwhile to be followed closely. Because intravital microscopy usually requires a fluorescent dye, its use has been restricted primarily to animal experiments. Some limited human applications of intravital microscopy have been developed (e.g. nailfold and skin capillaroscopy, see fig. 2) but they have not found widespread clinical acceptance [41]. These technologies have their place in the study of specific eye and skin conditions [40], the effects of drugs or the pathophysiology of some chronic diseases like diabetes mellitus [42]. They have not yet entered into routine perioperative and intensive care monitoring due to the cumbersome handling of the devices and the limited number of vascular beds that can be visualised.

\section{Orthogonal Polarisation Spectral (OPS) Imaging}

Recently OPS imaging has been introduced, which offers new clinically relevant perspectives for intravital microscopy in clinical practice since it allows for an easy, portable access to a variety of vascular beds [43]. Using the OPS imaging incorporated into the CYTOSCAN ${ }^{\mathrm{TM}}$ $\mathrm{A} / \mathrm{R}$ microvascular images can be obtained without the 


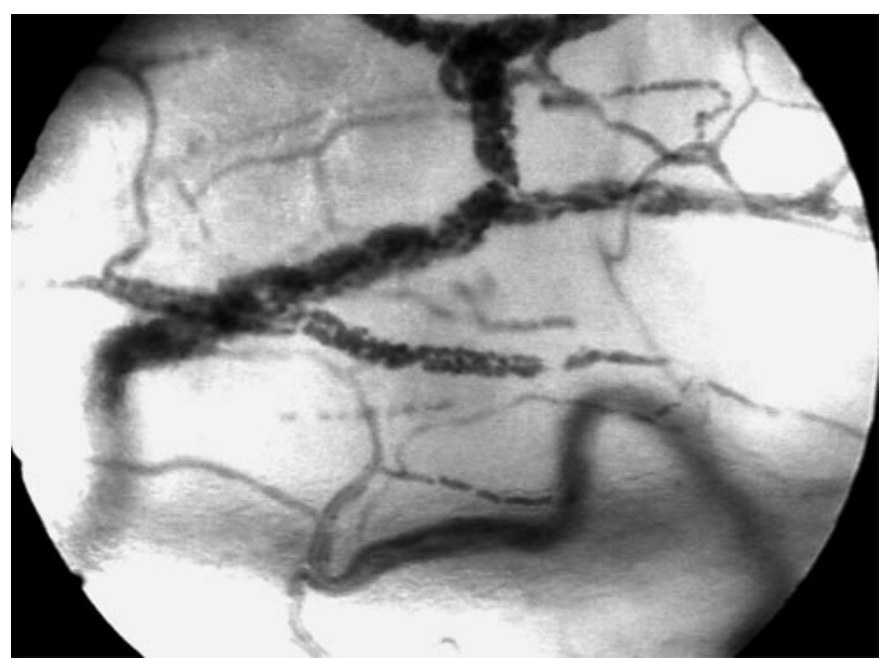

Fig. 3. OPS image taken from the human sublingual mucosa showing capillaries and post-capillary venules. The contrast is provided by the erythrocytes.

need for contrast enhancement with dyes. Microvascular perfusion of arterioles, capillaries and venules can be directly visualised and measured due to a 350-fold magnification (cf. fig. 3, image taken from the sublingual mucosa). Measurements of microvascular diameters, red blood cell velocity, and functional capillary density have been validated and compared with values obtained with intravital microscopy images using enhancing dyes [44]. Moreover in humans it has been shown that OPS imaging gives comparable results with measurements obtained by intravital microscopy images of nailfold capillaries [45].

OPS imaging has been used to study the microcirculation of many organs including the brain $[43,46]$, the liver [47], burn wounds [48] the gut and the skin of neonates. Genzel et al. [49] used OPS imaging to assess microvascular perfusion in neonates and found no differences in microvascular diameter and perfusion between term and preterm neonates. The latter, however, revealed a significant increase in red blood cell flow on the fifth day after birth which correlated with a decrease in hematocrit. The authors suggested that this technique can be used to assess impaired tissue perfusion, and currently conduct such an observational study in septic premature neonates.

Recently pathological microcirculation of brain tumours has been demonstrated with this technique and differences in the structure were revealed for meningiomas, glioblastomas and metastases [46]. The authors suggested that '... this technique could provide useful information about microcirculatory morphology and kinetics for diag-
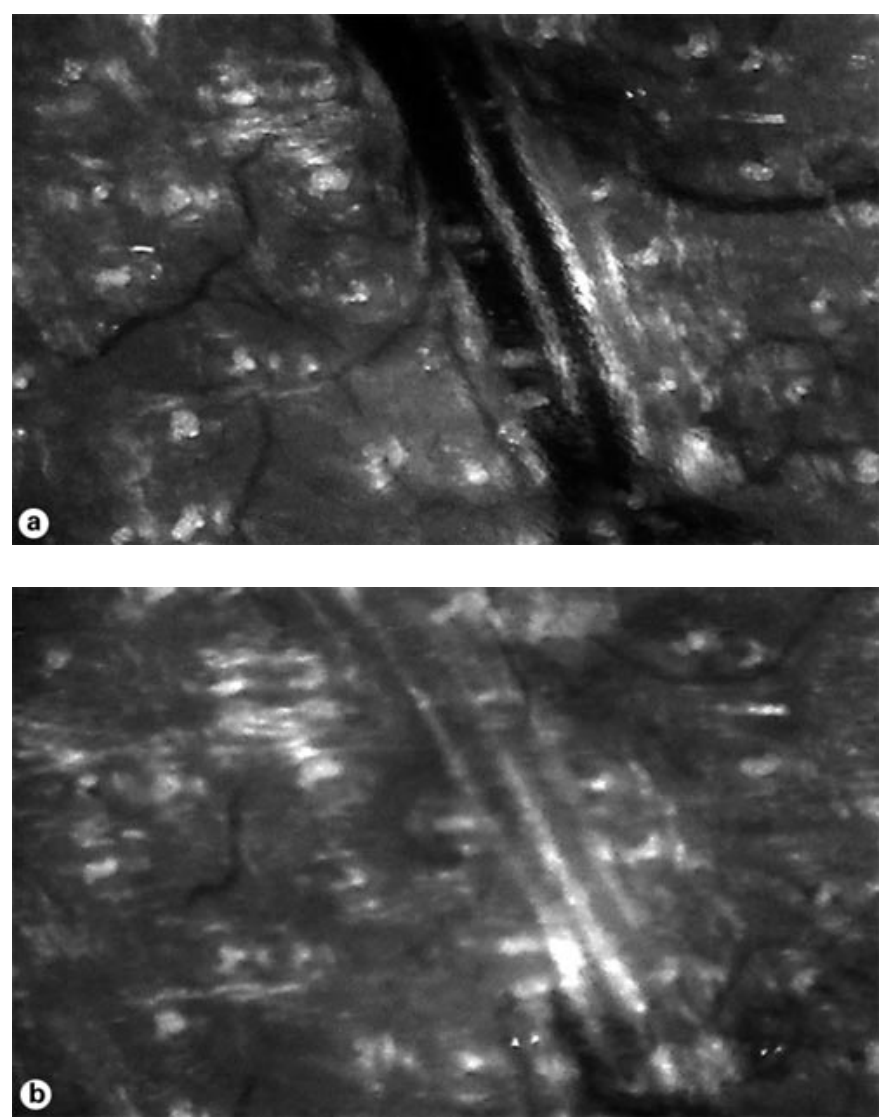

Fig. 4. a Post-capillary venules and capillaries in a beating pig heart prior to induction of regional ischemia using a monofilament tourniquet suture around the proximal ramus interventricularis anterior (RIVA). b The identical area of the heart during regional ischemia following the closure of the proximal RIVA using a tourniquet.

nostic use during neurosurgery'. Vollebregt et al. [50] found in pre-eclamptic women an impaired local skin microcirculation with OPS imaging, which was not detectable with LDF. OPS imaging allows to identify ischemic regions of the epicardium [51]. With a specific stabilising technique even images of the beating heart can be obtained (fig. $4 a, b$ ) and quantitative measurements of diameter and flow can be made.

To make clinical use of this technology a fast and easy to use analysis software is needed giving absolute values of microvascular perfusion. So far most clinical studies with OPS imaging have used a well validated but cumbersome manual analysis software routine [52]. A software tool developed in our group [53] enables the fast measurement of microvascular diameter, red cell velocity and flow as well as functional capillary density in a semi-automated fashion. Due to the clearly defined statistical mar- 
gins of this analysis it may be used in future for a fully automated bedside analysis of the OPS images.

Leukocytes labelled with acridine orange or rhodamine $6 \mathrm{G}$ can be visualised using fluorescent intravital microscopy [54]. This allows for quantitative assessment of the interaction of leukocytes with the endothelium. OPS imaging also allows for a visualisation of leukocytes where they appear as spacing within the red cell column. The typical slow rolling movement along the postcapillary venule can be identified. Using OPS imaging we regularly saw rolling and sticking leukocytes in images obtained during cardio-pulmonary bypass (CPB) as well as after strenuous exercise in healthy volunteers. We quantified these observation and could demonstrate a five-fold increase in the number of rolling leukocytes $1 \mathrm{~h}$ after termination of $\mathrm{CPB}$, which correlated with an increased expression of CD 18 and CD 65L [55]. We took those changes as an indication of ischemia-reperfusion injury since the arterial lactate concentration rose simultaneously. So far no adequate software exists to quantify the changes in rolling leukocytes which would be a prerequisite for a application parameter of patient monitoring.

This new technique, however, is also not free of methodological problems. The low depth of penetration $(5 \mathrm{~mm})$ and, thus, the limitation to superficial tissue, and movement and pressure artifacts affecting the obtained images have to be named. Hence, specific hard- and software have to be developed to allow a widespread use of this technology for assessment of microvascular perfusion at the bed side.

\section{Conclusion}

New optical methods for monitoring of the microcirculation have recently been developed offering a different approach to patient care. The well established technique of laser Doppler fluxmetry has recently been significantly improved overcoming some obstacles like spatial and temporal variation of erythrocyte flux. However, since no absolute values of red blood cell flux are available, a widespread clinical use seems currently unlikely. Near-infrared spectroscopy gives an index of microvascular hypoperfusion and some promising clinical application, e.g. for cerebral monitoring during carotid artery surgery. Due to a poor signal-to-noise ratio and some methodical flaws with respect to the tissue that is truly measured, perioperative and critical patient care have not adopted this technology. The clinical potential of intravital microscopy has recently been significantly enhanced due to the introduction of orthogonal polarisation spectral imaging. This new technique allows for an easy to use access to many vascular beds including the skin, mucous membranes, brain, heart and most visceral organs without the need for imaging-enhancing dyes. The true clinically value of this exciting technique has yet to be determined and will be depending on large clinical trials showing an impact on diagnostics and patient management.

\section{References}

1 Sibbald WJ, Messmer K, Fink MP: Roundtable Conference on Tissue Oxygenation in Acute Medicine, Brussels, Belgium, 14-16 March 1998. Intensive Care Med 2000;26:780-791.

2 Messmer K (ed): Ischemic Diseases and the Microcirculation. München, Zuckschwerdt, 1989.

3 Levy BI, Ambrosio G, Pries AR, Struijker-Boudier HA: Microcirculation in hypertension: A new target for treatment? Circulation 2001; 104:735-740.

4 Stern MD: In vivo evaluation of microcirculation by coherent light scattering. Nature 1975; 254:56-58.

5 Bonner R, Nossal R: Model for laser Doppler measurements of blood flow in tissue. Appl Opt 1980;20:2097-2107.

6 Tenland T, Salerud EG, Nilsson GE, Oberg PA: Spatial and temporal variations in human skin blood flow. Int J Microcirc Clin Exp 1983; 2:81-90.
7 Kernick DP, Tooke JE, Shore AC: The biological zero signal in laser Doppler fluximetry. Pflügers Arch 1999;437:624-631.

8 Braverman IM, Schechner JS, Silverman DG, Keh-Yen A: Topographic mapping of the cutaneous microcirculation using two outputs of laser-Doppler flowmetry: Flux and the concentration of moving blood cells. Microvasc Res 1992;44:33-48.

9 Wardell K, Jakobsson A, Nilsson GE: Laser Doppler perfusion imaging by dynamic light scattering. IEEE Trans Biomed Eng 1993;40: 309-316.

10 Gardner-Medwin JM, Taylor JY, Macdonald IA, Powell RJ: An investigation into variability in microvascular skin blood flow and the responses to transdermal delivery of acetylcholine at different sites in the forearm and hand. Br J Clin Pharmacol 1997;43:391-397.
11 Hahn M, Hahn C, Brauer K, Junger M: Skin thermoregulation during local cooling in healthy volunteers and patients with systemic sclerosis - synchronous assessment of capillary red blood cell velocity, laser Doppler flux and skin temperature. Vasa 1998;27:3-9.

12 Mayrovitz HN, Leedham JA: Laser-Doppler imaging of forearm skin: Perfusion features and dependence of the biological zero on heatinduced hyperemia. Microvasc Res 2001;62: 74-78.

13 Ubbink DT, Jacobs MJ, Tangelder GJ, Slaaf DW, Reneman RS: The usefulness of capillary microscopy, transcutaneous oximetry and laser Doppler fluxmetry in the assessment of the severity of lower limb ischaemia. Int J Microcirc Clin Exp 1994;14:34-44.

14 Belboul A, al Khaja N: The effect of protamine on the epicardial microflow and the graft flow in open-heart surgery. Perfusion 1997;12:99_ 106. 
15 Dodson TB, Neuenschwander MC, Bays RA: Intraoperative assessment of maxillary perfusion during le Fort I osteotomy. J Oral Maxillofac Surg 1994;52:827-831.

16 Buckley JG, Jones ML, Hill M, Sugar AW: An evaluation of the changes in maxillary pulpal blood flow associated with orthognathic surgery. Br J Orthod 1999;26:39-45.

17 Brauer K, Hahn M: Nonlinear analysis of blood flux in human vessels. Phys Med Biol 1999;44:1719-1733.

18 Arnold M, Witte H, Leger P, Boccalon H, Bertuglia S, Colantuoni A: Time-variant spectral analysis of LDF signals on the basis of multivariate autoregressive modelling. Technol Health Care 1999; 7:103-112.

19 Alexopoulos V, Kollias S, Leger P, Boccalon H, Csiki Z: Higher-order spectral analysis in laserDoppler flowmetry signal processing. Technol Health Care 1999;7:85-101.

20 Niazi ZB, Essex TJ, Papini R, Scott D, McLean NR, Black MJ: New laser Doppler scanner, a valuable adjunct in burn depth assessment. Burns 1993;19:485-489.

21 Droog EJ, Steenbergen W, Sjoberg F: Measurement of depth of burns by laser Doppler perfusion imaging. Burns 2001;27:561-568.

22 Ferrell WR, Balint PV, Egan CG, Lockhart JC, Sturrock RD: Metacarpophalangeal joints in rheumatoid arthritis: Laser Doppler imaging initial experience. Radiology 2001;220:257262.

23 Kernick DP, Shore AC: Characteristics of laser Doppler perfusion imaging in vitro and in vivo. Physiol Meas 2000;21:333-340.

24 Jöbsis FF: Noninvasive, infrared monitoring of cerebral and myocardial oxygen sufficiency and circulatory parameters. Science $1977 ; 198$ 1264-1267.

25 Rolfe P: Experience with invasive and noninvasive sensors for anaesthesia. Acta Anaesthesiol Scand Suppl 1995;104:61-68.

26 Faris F, Doyle M, Wickramasinghe Y, Houston R, Rolfe P, O'Brien S: A non-invasive optical technique for intrapartum fetal monitoring: Preliminary clinical studies. Med Eng Phys 1994;16:287-291.

27 Faris F, Rolfe P, Thorniley M, Wickramasinghe Y, Houston R, Doyle M, O'Brien S: Non-invasive optical monitoring of cerebral blood oxygenation in the foetus and newborn: Preliminary investigation. J Biomed Eng 1992; 14:303-306.

28 Wyatt JS, Cope M, Delpy DT, Wray S, Reynolds EO: Quantification of cerebral oxygenation and haemodynamics in sick newborn infants by near infrared spectrophotometry. Lancet 1986;2:1063-1066.
29 Edwards AD, Wyatt JS, Richardson C, Delpy DT, Cope M, Reynolds EO: Cotside measurement of cerebral blood flow in ill newborn infants by near infrared spectroscopy. Lancet 1988;2:770-771.

30 Germon TJ, Kane NM, Manara AR, Nelson RJ: Near-infrared spectroscopy in adults: Effects of extracranial ischaemia and intracranial hypoxia on estimation of cerebral oxygenation. Br J Anaesth 1994;73:503-506.

31 McCormick PW, Stewart M, Goetting MG, Dujovny M, Lewis G, Ausman JI: Noninvasive cerebral optical spectroscopy for monitoring cerebral oxygen delivery and hemodynamics. Crit Care Med 1991;19:89-97.

32 Pilkington SN, Hett DA, Pierce JM, Smith DC: Auditory evoked responses and near infrared spectroscopy during cardiac arrest. $\mathrm{Br} \mathrm{J}$ Anaesth 1995;74:717-719.

33 Kirkpatrick PJ, Smielewski P, Czosnyka M, Menon DK, Pickard JD: Near-infrared spectroscopy use in patients with head injury. $\mathrm{J}$ Neurosurg 1995;83:963-970.

34 Lassnigg A, Hiesmayr M, Keznickl P, Mullner T, Ehrlich M, Grubhofer G: Cerebral oxygenation during cardiopulmonary bypass measured by near-infrared spectroscopy: Effects of hemodilution, temperature, and flow. J Cardiothorac Vasc Anesth 1999;13:544-548.

35 Nollert G, Jonas RA, Reichart B: Optimizing cerebral oxygenation during cardiac surgery: A review of experimental and clinical investigations with near infrared spectrophotometry. Thorac Cardiovasc Surg 2000;48:247-253.

36 Nollert G, Shin'oka T, Jonas RA: Near-infrared spectrophotometry of the brain in cardiovascular surgery. Thorac Cardiovasc Surg 1998;46:167-175.

37 Kirkpatrick PJ, Smielewski P, Whitfield PC, Czosnyka M, Menon D, Pickard JD: An observational study of near-infrared spectroscopy during carotid endarterectomy. J Neurosurg 1995;82:756-763.

38 Terborg C, Schummer W, Albrecht M, Reinhart $\mathrm{K}$, Weiller $\mathrm{C}$, Rother J: Dysfunction of vasomotor reactivity in severe sepsis and septic shock. Intensive Care Med 2001;27:12311234.

39 Harris DN: Near infra-red spectroscopy. Anaesthesia 1995;50:1015-1016.

40 Fagrell B, Intaglietta M: Microcirculation: Its significance in clinical and molecular medicine. J Intern Med 1997;241:349-362.

41 Fagrell B: Advances in microcirculation network evaluation: An update. Int J Microcirc Clin Exp 1995;15(suppl 1):34-40.

42 Shore AC, Jaap AJ, Tooke JE: Capillary pressure in patients with NIDDM. Diabetes 1994; 43:1198-1202.
43 Groner W, Winkelman JW, Harris AG, Ince C, Bouma GJ, Messmer K, Nadeau RG: Orthogonal polarization spectral imaging: A new method for study of the microcirculation. Nat Med 2000;5:1209-1213.

44 Harris AG, Sinitsina I, Messmer K: The Cytoscan ${ }^{\mathrm{TM}}$ Model E-II, a new reflectance microscope for intravital microscopy: Comparison with the standard fluorescence method. J Vasc Res 2000;37:469-476.

45 Mathura KR, Vollebregt KC, Boer K, De Graaff JC, Ubbink DT, Ince C: Comparison of OPS imaging and conventional capillary microscopy to study the human microcirculation. J Appl Physiol 2001;91:74-78.

46 Mathura KR, Bouma GJ, Ince C: Abnormal microcirculation in brain tumours during surgery. Lancet 2001;358:1698-1699.

47 Langer S, Harris AG, Biberthaler P, von Dobschuetz E, Messmer K: Orthogonal polarization spectral imaging as a tool for the assessment of hepatic microcirculation: A validation study. Transplantation 2001;71:1249-1256.

48 Langer S, Hatz R, Harris AG, HernandezRichter T, Maiwald G, Messmer K: Assessing the microcirculation in a burn wound by use of OPS imaging. Eur J Med Res 2001;6:231-234.

49 Genzel-Boroviczeny O, Strötgen J, Harris AG Christ F: Orthogonal polarization spectral imaging (OPS): A novel method to measure transcutaneously the microcirculation in term and preterm infants. Pediatr Res 2002 (in press).

50 Vollebregt KC, Boer K, Mathura KR, De Graaff JC, Ubbink DT, Ince C: Impaired vascular function in women with pre-eclampsia observed with orthogonal polarisation spectral imaging. BJOG 2001;108:1148-1153.

51 Boehm DH, Christ F, Harris AG, Reichenspurner H, Reichart B, Messmer K: First application of intravital microscopy on the beating heart using OPS imaging (abstract). J Vasc Res 2000;37:23

52 Klyscz T, Junger M, Jung F, Zeintl H: Cap image - a newly-developed computer-aided videoframe analysis system for dynamic capillaroscopy. Biomed Tech 2000;42:168-175.

53 Schaudig S, Dadasch B, Kellam K, Christ F: Validation of an analysis software for OPS imaging used in humans (abstract). Proc 7th World Congr Microcirc 2001, pp 2-59.

54 Nolte D, Lehr HA, Messmer K: Adenosine inhibits postischemic leukocyte-endothelium interaction in postcapillary venules of the hamster. Am J Physiol 1991;261:H651-H655.

55 Schaudig S, Schiessler C, Harris AG, Reichenspurner H, Messmer K, Christ F: Intravital microscopy in humans following circulatory arrest for surgery of the ascending aorta. J Vasc Res 2002 (in press). 